\title{
Ambiente térmico y bienestar psicológico en viviendas de interés social en clima cálido seco
}

\section{Thermal environment and psychological well-being in low prices housing on warm and dry climate}

\author{
Noelia Beatriz Espinoza Gallego \\ Daniel González Lomelí \\ José Manuel Ochoa de la Torre \\ Víctor Corral Verdugo \\ Universidad de Sonora
}

\section{Resumen}

El objetivo del presente estudio fue conocer el efecto que tiene el ambiente térmico de las viviendas de interés social en el bienestar psicológico de sus residentes durante dos estaciones del año (verano e invierno), a partir de desarrollar y probar dos modelos de ecuaciones estructurales. El estudio se llevó a cabo en la ciudad de Hermosillo, Sonora, México, la cual tiene como característica su tipo de clima: muy árido, cálido o semidesértico. El estudio de la percepción ambiental involucra claramente dos aspectos abordados en este trabajo: un componente objetivo o físico y un componente subjetivo. El primero representado por las características del entorno físico, las cuales inciden en el significado que a él se le atribuyen, y el segundo, por un conjunto de representaciones internas que el sujeto hace de su entorno. Se realizó esta evaluación considerando el componente físico mediante la utilización de instrumentos para calcular las condiciones térmicas de las viviendas y el segundo, mediante la escala de bienestar psicológico asociado a la vivienda, instrumento adaptado y validado para este trabajo. Como principal hallazgo podemos mencionar que los modelos obtenidos mostraron que el ambiente térmico al interior de la vivienda afectó el bienestar psicológico de las personas sólo en la temporada de verano, ya que se observó una relación negativa y significativa entre ambos factores y no se presentó esta relación significativa en el modelo desarrollado para el invierno.

Palabras clave: ambiente térmico, bienestar psicológico, viviendas de interés social, habitabilidad, percepción ambiental.

Nota del autor

Noelia Beatriz Espinoza Gallego, Doctorado en Humanidades, Universidad de Sonora (UNISON); Daniel González Lomelí, Posgrado Integral en Ciencias Sociales, UNISON; José Manuel Ochoa de la Torre, Departamento de Arquitectura y Diseño, UNISON; Víctor Corral Verdugo, Posgrado Integral en Ciencias Sociales, UNISON.

Esta investigación fue subvencionada por el Consejo Nacional de Ciencia y Tecnología (CONACYT) de México a través de la Beca de Posgrado de Excelencia 234798.

La correspondencia en relación con este artículo debe dirigirse a Noelia Beatriz Espinoza Gallego, Doctorado en Humanidades, UNISON, bulevar Luis Encinas y Rosales s/n, colonia Centro, C.P. 83000, Hermosillo Sonora, México.

Correo electrónico: noeliabeatriz9@hotmail.com 


\begin{abstract}
The objective of this study was to determine the effect of the thermal environment of social housing in the psychological well-being of its residents in two seasons (summer and winter), starting to develop and test two structural equation models. The study was conducted in Hermosillo, Sonora, Mexico, a city located in a semi desert, arid and warm climate. The study of meaning and environmental perception involves two aspects included in this job: One is an objective or physical component, and the second one is a subjective component. The first one was indicated by features of the physical context, which affect the meaning attributed to it, and the second, one by a set of internal representations about the environment including the emotions produced experienced by people. An evaluation was conducted considering the above-mentioned components. The first one was evaluated using tools that assessed the housing thermal conditions, and the second one through an instrument adapted and validated for this study with the purpose of assessing aspects related to psychological well-being. The main finding we mention that the obtained models showed that the thermal environment inside the housing affects the psychological well-being of people only in the summer season as a negative and significant relationship between the two factors is observed and not this relationship is presented significant in the model developed for the winter.

Keywords: thermal environmental, psychological well-being, social housing, habitability, environmental perception.
\end{abstract}

Uno de los derechos humanos es el derecho universal a una vivienda, digna y adecuada, este aparece recogido en el Artículo veinticinco de la Declaración Universal de los Derechos Humanos y en el Artículo once del Pacto Internacional de Derechos Económicos, Sociales y Culturales (PIDESC). En el ámbito internacional, el acceso a una vivienda adecuada forma parte de la agenda de la Organización de las Naciones Unidas (ONU), éste ha sido considerado entre los Objetivos de Desarrollo del Milenio (2000). Como tal, dicha disposición se suma a los parámetros y acciones ejecutadas en búsqueda de una mejor calidad de vida, al reiterar la necesaria atención del hábitat para el desarrollo humano.

\section{Vivienda y habitabilidad}

La vivienda ocupa un papel central en la calidad de vida de los individuos y su posibilidad de acceso a otros factores y servicios que permiten niveles mínimos de bienestar (Ponce, 2011). La preocupación por el conocimiento de las condiciones habitacionales y la carencia de vivienda ha permitido, también, desarrollos metodológicos que evidencian una gran variedad de situaciones. Este déficit de vivienda incluye situaciones que van desde la inadecuación de los materiales, la sobreocupación de espacios, la ausencia de conexión a servicios básicos, su localización en zonas de riesgo, hasta las demás facetas que la perspectiva del hábitat plantea como componentes ineludibles de toda intervención (Cuervo, 2012) . 


\section{Comportamiento y medio ambiente}

Rara vez uno se detiene a pensar cómo afecta a nuestras vidas el medio ambiente en el que vivimos, estudiamos, trabajamos. Sin embargo, los escenarios que rodean y sustentan nuestra vida diaria, ejercen una enorme influencia en la manera en que pensamos, nos sentimos y, por supuesto, cómo nos comportamos. Por ejemplo, una persona que despierta en una habitación agradable, bien iluminada, térmicamente confortable, probablemente comenzará su día con mayor optimismo que otra cuyo dormitorio es pequeño, ruidoso, poco ventilado, con muebles incómodos, etcétera.

Lewin (1964) fue uno de los primeros psicólogos en otorgarle la verdadera importancia a la relación entre los seres humanos y el medio ambiente. Su objeto de estudio se centró en determinar el tipo de influencia que ejercía el espacio físico sobre las personas y la clase de relaciones que éstas establecían a su vez con el mismo. El autor señalaba básicamente tres tipos de relaciones: primero, el ser humano es capaz de modificar el ambiente que lo rodea; segunda, es necesario considerar al individuo y el medio ambiente como una entidad única e inseparable; y tercera, así como el sujeto actúa sobre el medio ambiente, el medio ambiente también puede afectar a la persona de manera profunda.

A partir de la década de los cincuenta, la psicología empezó a poner atención en las formas cómo el ambiente físico configura la vida de las personas, surgió así un nuevo e interesante campo de estudio: la psicología ambiental. En la actualidad, existen muchas definiciones para esta área de estudio, la mayoría de los autores coincide con la idea de que ésta estudia las relaciones recíprocas entre la conducta humana y el mundo social y físico en el que viven las personas (Aragonés \& Amérigo, 2000).

Corral (2010) señala que, de acuerdo con esta definición, el interés de esta área se centra en los efectos que tiene la conducta humana en el ambiente y viceversa, de tal manera que siempre que se da una interacción entre la persona y el entorno, ambos se afectan mutuamente. El mismo autor, nos dice que históricamente, la psicología ambiental se ha dividido en dos vertientes: por una parte, la psicología de la conservación ambiental, enfocada en el impacto del comportamiento en entornos naturales y las repercusiones del cuidado del medio; por otra, la denominada psicología arquitectónica, donde se pretende enmarcar este proyecto, en relación con los efectos que tiene el diseño y los ambientes construidos en la conducta.

En congruencia con esta segunda vertiente, planteada por Corral (2010) y Amérigo (1995), se menciona la importancia que tiene dentro del ámbito de la psicología ambiental el estudio de los espacios construidos; se señala que las relaciones establecidas entre el comportamiento y aquel ambiente donde la mano del hombre ha intervenido construyéndolo o modificándolo, ha despertado un especial interés en los investigadores y que los trabajos realizados en esta temática, no sólo tratan de investigar la medida en la cual el comportamiento afecta el entorno 
construido, sino, que de una manera biunívoca, se estudia la manera en que éste puede influir en la conducta humana. En dicho sentido, Holahan (2010) enfatiza la importancia de la participación interdisciplinaria en esta área, al señalar que el estudio de la conducta humana en ambientes físicos requiere del trabajo de investigadores en ciencias sociales y de arquitectos y responsables de los diseños de los ambientes humanos.

Existe una gran cantidad de esfuerzos en investigación para el estudio del impacto de los factores medio ambientales en el comportamiento de las personas. Muchos estudios han aportado evidencia de que el ambiente térmico puede tener un efecto en la salud y en las emociones de las personas. A continuación se presenta una serie de estudios en relación con el tema.

Cassidy (1997) nos habla del impacto que pueden llegar a tener sobre la vida emocional de las personas los factores del medio ambiente como el ruido, olor, luz y temperatura o clima, haciendo énfasis en los efectos negativos que éstos pueden llegar a tener tanto en la salud física y mental de las personas como en las relaciones que establecen con otras personas.

Rosen (1985) sugiere que hay una correlación entre la presión sanguínea producida por el clima y los dolores de cabeza, migraña y cambios en los estados de ánimo. Rim (1975) encontró que durante los períodos de viento en el desierto de Israel, se podían observar incrementos en las conductas neuróticas y de extroversión; así como decrementos en los niveles del coeficiente intelectual de los sujetos estudiados.

En los años setenta, uno de los más prolíficos investigadores en el área de estudio de la temperatura y la emoción fue Baron (1972, 1978), él argumentaba que los climas cálidos propiciaban el incremento del potencial para las conductas agresivas y que, en general, se presentaba una excitación en las respuestas emocionales de las personas. En esta misma línea podemos encontrar los estudios de Bell y Fusco (1989), Cohn (1993) y Field (1992), quienes encontraron correlaciones positivas con muchos tipos de crímenes violentos, como asesinatos, violaciones, asaltos y violencia intrafamiliar.

Bell y Greene (1982) aluden a la importancia del estudio de las capacidades humanas para tolerar temperaturas extremas; realizaron trabajos sobre la percepción de la temperatura y los mecanismos psicológicos involucrados en la termorregulación, estudiando el estrés térmico, contrastándolo con los efectos sociales en ambientes cálidos y fríos.

Algunos datos permiten afirmar que comúnmente durante los meses de invierno las personas incrementan sus niveles de ansiedad al tiempo que se encuentran más expuestas a padecer depresión (Rosenthal, 1993; Rohan et al., 2003, citados en Cohen-Sánchez, 2009). Esto frecuentemente se relaciona con el bajo nivel de luz en el ambiente y el corto fotoperíodo de estos meses (Persinger, 1980; Watson, 2000, citados en Keller et al., 2005). 
Lambert, Reid, Kaye, Jennings y Esler (2003) examinaron la frecuencia de suicidio y su relación con factores meteorológicos en el estado de Victoria, entre enero de 1990 y abril de 1999. Encontraron que la incidencia de suicidio, específicamente el suicidio violento, tuvo su punto más bajo en el invierno y su apogeo en la primavera y el verano. Este estudio concluye que la incidencia de suicidio en el sureste de Australia muestra un claro patrón estacional, ya que presenta una correlación positiva con los niveles de prevalencia de luz solar.

También se han realizado estudios para medir el posible impacto del ambiente térmico en el desempeño escolar. Hernández y Gómez (2007) llevaron a cabo un investigación en la ciudad de Colima en México, ellos seleccionaron tres escuelas con base en los resultados de sus indicadores académicos y constituyeron dos grupos, uno de escuelas que no presentaban problemas académicos graves y otro de escuelas con un menor desempeño, todos grupos del turno vespertino a fin de analizar condiciones de ambientes menos favorables. Se llevó a cabo un monitoreo de la temperatura en tres temporadas climáticas representativas para esta ciudad en aulas que fueron seleccionadas conforme con su ubicación y el objetivo de diferenciar el grado de influencia de la irradiación solar o circulación de los vientos. Contrario a la correlación positiva que se esperaría encontrar, es decir, a mayor ambiente térmico, mayor aprovechamiento escolar, los estudiantes mostraron un mejor desempeño durante la temporada de temperaturas extremas o cálidas que cuando las condiciones se acercaban a la temperatura de neutralidad para esta ciudad.

El estudio sugiere la posibilidad de que el malestar térmico frente a la comodidad sea un componente de la felicidad de un país, pues el estrés generado, ya sea por frío o por calor, mina el sentimiento de felicidad en los países pobres; mientras que en los países ricos lo consolida. Esto nos indica que la gente es más feliz en la medida en que tiene la certidumbre de tener los medios económicos para poder hacer frente a las adversidades del medio ambiente, y proveer, a ellos mismos y a su familia, de condiciones de vivienda dignas y confortables; en contraste con la incertidumbre generada en los países pobres, donde no se tienen los recursos para enfrentar las mismas condiciones y, según este estudio, es una fuente de infelicidad.

\section{Objetivo general de la investigación}

Conocer el efecto de las condiciones térmicas de las viviendas en el bienestar psicológico de sus residentes en dos estaciones del año, a partir de modelos de ecuaciones estructurales.

\section{Objetivos específicos}

1. Adaptar un instrumento para la evaluación del bienestar psicológico asociado a la vivienda y determinar sus propiedades psicométricas de consistencia y estructura factorial, además de la descripción de la distribución de la escala de bienestar psicológico.

2. Poner a prueba un modelo de relaciones directas e indirectas entre las condiciones térmicas de las viviendas y el bienestar psicológico de sus residentes en la temporada de verano. 
3. Poner a prueba un modelo de relaciones directas e indirectas entre las condiciones térmicas de las viviendas y el bienestar psicológico de sus residentes en la temporada de invierno.

4. Realizar un análisis comparativo de los datos obtenidos para las temporadas de invierno y verano.

\section{Preguntas de investigación}

1. ¿Cómo evalúan el ambiente térmico del interior de sus viviendas, los residentes de viviendas de interés social construidas en clima cálido-seco?

2. ¿Qué tipo de relación existe entre el bienestar psicológico y la sensación térmica reportada por los residentes de viviendas de interés social?

\section{Hipótesis del estudio}

El ambiente físico térmico se relaciona de manera diferente con el bienestar psicológico de las personas dependiendo de la estación del año.

\section{Método}

\section{Participantes}

Los participantes del presente estudio fueron 130 residentes de viviendas de interés social, de éstos 97 eran mujeres; sus edades oscilaban entre $\operatorname{los} 18$ y 73 años, siendo la media de edad 32.6 años. El tipo de muestra fue de tipo probabilístico, para establecerla se obtuvieron los planos del fraccionamiento donde se llevaría a cabo el levantamiento de datos con el fin de seleccionar las viviendas que se visitarían. El método empleado fue un muestreo aleatorio simple.

\section{Instrumentos}

Para el levantamiento de datos se utilizaron los siguientes instrumentos:

1. Una plantilla con variables sociodemográficas y condiciones físicas y de equipamiento de la vivienda.

2. Plantilla para el registro de datos de monitoreo físico de temperatura de bulbo seco, humedad relativa, velocidad del viento.

3. Escala de percepción del ambiente al interior de la vivienda, conformada por ocho subescalas de medida de categorías ordinadas, las cuales se describen a continuación:

1) Escala de percepción de sensación térmica personal constituida por una escala simétrica de dos polos, comprende un punto central de indiferencia y dos veces tres grados de intensidad creciente, que va de $-3=$ mucho frío, un punto de indiferencia que es $0=$ ni calor ni frío y $3=$ mucho calor.

2) Escala de percepción de sensación de humedad constituida por una escala simétrica de dos polos, comprende un punto central de indiferencia y dos veces tres grados de intensidad creciente, que va de $-3=$ muy húmedo, un punto de indiferencia que es $0=$ normal y $3=$ muy seco.

3) Escala de percepción de sensación nocturna de temperatura constituida por una escala simétrica de dos polos, comprende un punto central de indiferencia y dos veces tres grados de intensidad creciente, que va de $-3=$ mucho frío, un punto de indiferencia que es $0=$ ni calor ni frío y 3 = mucho calor. 
4) Escala de preferencia de temperatura constituida por una escala simétrica de dos polos, comprende un punto central de indiferencia y dos veces tres grados de intensidad creciente, que va de $-3=$ mucho más fresco, un punto de indiferencia que es $0=\sin$ cambio y $3=$ mucho más caluroso.

5) Escala de preferencia de ventilación constituida por una escala simétrica de dos polos, comprende un punto central de indiferencia y dos veces un grado de intensidad creciente, que va de $-1=$ preferencia de más ventilación, un punto de indiferencia que es $0=\sin$ cambio y 1 $=$ preferencia de menos ventilación.

6) Escala evaluativa de sensación de ventilación constituida por una escala de cuatro grados y un polo, con un punto de origen indicando la ausencia de efecto y 3 grados de intensidad creciente, que va de $0=$ ninguna ventilación a 4 $=$ mucha ventilación.

7) Escala de declaración personal de aceptabilidad, donde se expresa la aceptación o rechazo del ambiente térmico.

8) Escala de declaración personal de tolerancia del ambiente térmico.

4. Escala de bienestar psicológico asociado a la vivienda, adaptada y validada para este estudio, a partir de dos escalas de una adaptación española de las escalas de bienestar psicológico de Carol Ryff (Díaz et at., 2006): a) relaciones positivas con otras personas $y$ b) dominio del entorno y dos dimensiones de la escala de bienestar psicológico de Sánchez
(1998): a) bienestar subjetivo y b) bienestar material. Posteriormente, se adaptó la redacción de los reactivos originales con el fin de adecuarlos al contexto de la vivienda, teniendo en cuenta criterios de claridad y ajuste a la definición de cada una de las escalas. Cabe mencionar que los reactivos se adaptaron y crearon haciendo referencia a situaciones relacionadas con el contexto de vivienda (usuarios, espacio habitable, materiales $\mathrm{y}$ equipamiento, además de barrio y vecinos); se generó un mínimo de cuatro reactivos por cada una de ellas.

El instrumento fue aplicado en su versión preliminar a una muestra de 27 personas, la cual se llevó a cabo de manera individual, solicitando a los entrevistados que hicieran comentarios en cuanto a la redacción de los reactivos, las instrucciones, del formato de presentación de la prueba, complejidad y estructura del instrumento en general. Se reportaron sugerencias de modificaciones a la prueba, las cuales fueron atendidas llevando a cabo los ajustes necesarios de aquellos ítems que durante la aplicación generaron confusión.

Una vez realizados los ajustes necesarios arrojados por el estudio preliminar, se implementó un estudio piloto con el fin de analizar las propiedades psicométricas de la escala adaptada; los resultados se describen en el apartado de resultados de este documento.

El instrumento final quedó conformado por 36 reactivos, el formato de respuesta que 
se utilizó tiene puntuaciones comprendidas entre 5 (totalmente de acuerdo) y 1 (totalmente en desacuerdo), los ítems están divididos en cuatro escalas. Los reactivos de cada escala hacen referencia a situaciones. La primera "Dominio del entorno" consta de quince reactivos, de los cuales, dos de ellos hacen referencia a los materiales de construcción y el espacio habitable; siete al barrio que habitan los encuestados; y seis a las relaciones que establecen los encuestados con sus vecinos. La escala "Relaciones personales" consta de diez reactivos, tres hacen referencia a los usuarios; tres más a los materiales de construcción y el espacio habitable; y cuatro las relaciones que establecen los encuestados con sus vecinos. La escala "Bienestar subjetivo" consta de seis reactivos, cinco hacen referencia al usuario y uno al barrio. Por último, la escala "Bienestar material" está conformada por quince reactivos, de los cuales, tres hacen referencia a los usuarios; diez a los materiales de construcción y el espacio habitable; uno al barrio que habitan los encuestados; y uno a las relaciones que establecen los encuestados con sus vecinos.

\section{Procedimiento}

La recogida de datos se efectuó a cabo de manera directa e individual; se acudió a los domicilios seleccionados, se solicitó su consentimiento para participar, explicándoles a las personas el objetivo del estudio y se les informó que éste se llevaría a cabo en dos etapas. Con el fin de recabar los datos se realizaron dos mediciones del ambiente térmico al interior de la vivienda y se aplicó a los usuarios la escala de bienestar psicológico asociado a la vivienda en dos ocasiones, para la temporada de verano durante los meses de julio y agosto de 2012 y para la temporada invernal en enero y febrero de 2013. El tiempo de aplicación fue de aproximadamente 25 minutos en cada domicilio.

\section{Análisis de datos}

Para el análisis de los datos se empleó la técnica de modelamiento de ecuaciones estructurales (MEE). El MEE es una técnica de análisis estadístico multivariado utilizada para contrastar modelos que proponen relaciones causales entre las variables, es decir, permiten estimar el efecto y las relaciones entre múltiples variables. La gran ventaja de este tipo de modelos es que permiten proponer el tipo y dirección de las relaciones esperadas entre las diversas variables contenidas en él, para posteriormente, estimar los parámetros que vienen especificados por las relaciones propuestas a nivel teórico.

Por este motivo se denominan también modelos confirmatorios, ya que el interés fundamental es "confirmar" mediante el análisis de la muestra las relaciones propuestas a partir de la teoría explicativa que se haya decidido utilizar como referencia. Debido a que se trata de un modelo de tipo estadístico, contempla la existencia de errores de medida en las observaciones obtenidas de la realidad; habitualmente, incluyen múltiples variables observables y múltiples variables no observables (latentes). 
El establecimiento de relaciones causales entre variables constituye un aspecto fundamental de la investigación científica. Es difícil o resulta poco ético llevar a cabo en condiciones experimentales estudios que pretendan medir el impacto que puede tener el ambiente térmico en el comportamiento de las personas.

En este sentido, el MEE se presenta como una técnica útil para poder medir objetivamente la relación entre el ambiente físico térmico y el bienestar psicológico, así como también para encontrar la magnitud de la influencia que el ambiente térmico puede ejercer en el bienestar psicológico de una persona y viceversa. Para este fin, se empleó el paquete estadístico EQS 6.1 .

\section{Resultados}

En este apartado se muestran los resultados obtenidos en: a) el estudio piloto realizado para determinar las propiedades psicométricas de consistencia y estructura factorial, además de la descripción de la distribución de la escala de bienestar psicológico y b) los modelos de ecuaciones estructurales de ambiente térmico y bienestar psicológico asociado a la vivienda.

\section{Resultados del estudio piloto para determi-} nar las propiedades psicométricas de la escala de bienestar psicológico

La escala de bienestar psicológico asociado a la vivienda fue construida y validada en un estudio previo a esta investigación, el método para la extracción fue el de rotación Promax y el criterio para determinar el número de factores fue exploratorio, sin determinar el número de factores a extraer. Se tomó como criterio de aceptación que los pesos factoriales de cada uno de los reactivos fuesen de 0.40 o más, que tuvieran una carga positiva y que quedaran integrados al menos por tres reactivos. Las tablas 1, 2, 3 y 4 muestran los resultados del análisis factorial exploratorio, se observa que los factores se construyeron de manera coherente, los pesos factoriales altos y significativos $(p>$ $.05)$ se consideraron indicadores de validez de constructo convergente para cada uno de ellos.

El estadístico alfa de Cronbach se utilizó para analizar la consistencia interna de las escalas. Se reportaron los índices de confiabilidad para cada una de las escalas, así como las medias y desviación estándar para cada uno de sus reactivos. La escala Dominio del entorno tuvo un alfa de .81 con una puntuación media de 2.4 (tabla 1); la escala Relaciones personales obtuvo un valor de alfa igual a .84 con una puntuación media de 2.7 (tabla 2); la escala Bienestar subjetivo resultó con un valor de $.90 \mathrm{y}$ una puntuación media de 1.8 (tabla 3 ) y la escala Bienestar material obtuvo un valor de alfa de .90 con una puntuación media de 2.1 (tabla 4). 


\section{Tabla 1}

Pesos factoriales y análisis de confiabilidad (consistencia interna) de la escala Dominio del entorno

\begin{tabular}{|c|c|c|c|c|c|c|}
\hline Escala/reactivos & Mínimo & Máximo & Media & $\mathrm{DE}$ & $\mathrm{PF}^{*}$ & Alfa** \\
\hline Dominio del entorno & 1 & 5 & 2.40 & & & .81 \\
\hline $\begin{array}{l}\text { 1. He sido capaz de adquirir una vivienda en el } \\
\text { barrio que me gusta. }\end{array}$ & & & 2.26 & 1.172 & .55 & \\
\hline $\begin{array}{l}\text { 4. Si tuviera que elegir de nuevo un lugar donde } \\
\text { vivir elegiría el mismo fraccionamiento. }\end{array}$ & & & 2.36 & 1.230 & .48 & \\
\hline $\begin{array}{l}\text { 5. Me siento seguro en el fraccionamiento en el } \\
\text { que vivo }\end{array}$ & & & 2.35 & 1.126 & .59 & \\
\hline $\begin{array}{l}\text { 6. En mi barrio puedo confiar en la mayoría de } \\
\text { la gente. }\end{array}$ & & & 2.70 & 1.307 & .47 & \\
\hline $\begin{array}{l}\text { 7. Puedo decir que soy afortunado por tener la } \\
\text { casa que tengo. }\end{array}$ & & & 1.86 & 1.035 & .82 & \\
\hline $\begin{array}{l}\text { 8. El lugar en el que vivo me hace sentir seguro } \\
\text { por lo tanto me siento tranquilo. }\end{array}$ & & & 2.50 & 1.205 & .84 & \\
\hline $\begin{array}{l}\text { 9. Creo que mi barrio es un buen lugar para que } \\
\text { mis hijos crezcan. }\end{array}$ & & & 2.51 & 1.137 & .81 & \\
\hline $\begin{array}{l}\text { 14. Mi casa está mejor equipada que la de mis } \\
\text { vecinos }\end{array}$ & & & 2.68 & 1.086 & .46 & \\
\hline $\begin{array}{l}\text { 15. Me gusta convivir con mis vecinos al hacer } \\
\text { uso de los espacios recreativos (parques, pla- } \\
\text { zas, espacios deportivos) que hay cerca de mi } \\
\text { casa. }\end{array}$ & & & 2.52 & 1.145 & .60 & \\
\hline
\end{tabular}

*Pesos factoriales para cada reactivo de la escala.

${ }^{* *}$ Alfa de Cronbach fue utilizado como indicador de confiabilidad. 
Tabla 2

Pesos factoriales y análisis de confiabilidad (consistencia interna) de la escala Relaciones personales

\begin{tabular}{|c|c|c|c|c|c|c|}
\hline Escala/reactivos & Mínimo & Máximo & Media & $\mathrm{DE}$ & $P F^{*}$ & Alfa** \\
\hline Relaciones personales & 1 & 5 & 2.70 & & & .84 \\
\hline $\begin{array}{l}\text { 1. A menudo me siento solo en mi casa y siento } \\
\text { que no puedo compartir mis preocupaciones con } \\
\text { los miembros de mi familia. }\end{array}$ & & & 3.04 & .348 & .58 & \\
\hline $\begin{array}{l}\text { 2. En general, siento que en mi casa no todos } \\
\text { quieren escucharme cuando necesito hablar. }\end{array}$ & & & 2.91 & 1.365 & .69 & \\
\hline $\begin{array}{l}\text { 3. Sé que puedo confiar en mi familia (personas } \\
\text { que viven en mi casa) y ellos saben que puedo } \\
\text { confiar en mí. }\end{array}$ & & & 1.94 & 1.301 & .66 & \\
\hline $\begin{array}{l}\text { 4. Percibo mi casa como un espacio agradable } \\
\text { que promueve armonía entre las personas que la } \\
\text { habitan. }\end{array}$ & & & 2.05 & 1.178 & .65 & \\
\hline $\begin{array}{l}\text { 5. Disfruto cuando recibo amigos en mi casa } \\
\text { porque sé que es un lugar agradable (tempera- } \\
\text { tura). }\end{array}$ & & & 2.23 & 1.240 & .51 & \\
\hline $\begin{array}{l}\text { 6. Creo que la gente que me visita realmente } \\
\text { disfruta cuando llega a mi casa. }\end{array}$ & & & 2.57 & 1.289 & .79 & \\
\hline $\begin{array}{l}\text { 7. Me parece que la mayor parte de las personas } \\
\text { que conozco tiene más y mejores relaciones con } \\
\text { sus vecinos que yo. }\end{array}$ & & & 3.46 & .985 & .47 & \\
\hline $\begin{array}{l}\text { 8. No he logrado experimentar muchas rela- } \\
\text { ciones cercanas y de confianza con mis vecinos. }\end{array}$ & & & 3.48 & .923 & .49 & \\
\hline
\end{tabular}

*Pesos factoriales para cada reactivo de la escala.

${ }^{* *}$ Alfa de Cronbach fue utilizado como indicador de confiabilidad.

Tabla 3

Pesos factoriales y análisis de confiabilidad (consistencia interna) de la escala Bienestar subjetivo

\begin{tabular}{|c|c|c|c|c|c|c|}
\hline Escala/reactivos & Mínimo & Máximo & Media & $\mathrm{DE}$ & $\mathrm{PF}^{*}$ & Alfa** \\
\hline Bienestar subjetivo & 1 & 5 & 1.80 & & & .90 \\
\hline 1. Me gusta divertirme. & & & 1.64 & .980 & .67 & \\
\hline 2. Me siento jovial. & & & 1.92 & 1.041 & .71 & \\
\hline $\begin{array}{l}\text { 3. En mi casa yo duermo bien y de forma } \\
\text { tranquila. }\end{array}$ & & & 1.79 & .977 & .69 & \\
\hline $\begin{array}{l}\text { 4. Creo que soy útil y necesario a la gente que } \\
\text { me rodea. }\end{array}$ & & & 1.76 & 1.036 & .92 & \\
\hline 5. Creo que me sucederán cosas agradables. & & & 1.79 & 1.122 & .90 & \\
\hline
\end{tabular}

${ }^{*}$ Pesos factoriales para cada reactivo de la escala.

${ }^{* *}$ Alfa de Cronbach fue utilizado como indicador de confiabilidad. 
Tabla 4

Pesos factoriales y análisis de confiabilidad (consistencia interna) de la escala Bienestar material

Escala/reactivos

Bienestar material

$\begin{array}{llllll}\text { Mínimo } & \text { Máximo } & \text { Media } & \mathrm{DE} & \mathrm{PF}^{*} & \text { Alfa** }^{*} \\ 1 & 5 & 2.10 & & & .90\end{array}$

1. He construido un hogar y un estilo de vida a

$2.06 \quad 1.144 \quad .40$

mi gusto.

2. Siento que mi casa ofrece ambientes

$1.74 \quad .949 \quad .50$

agradables y los disfruto con mi familia (personas que viven conmigo).

3. Cuando pienso en lo que he logrado en relación al equipamiento de mi casa me siento $\begin{array}{lll}1.95 & .925 \quad .51\end{array}$ optimista.

4. Siento que mi casa está equipada y que

$1.92 \quad .981 \quad .61$
tiene todo lo que necesito para poder vivir dignamente.

5. Estoy conforme con mi casa tal y como está.

$\begin{array}{lll}2.39 & 1.222 \quad .49\end{array}$

6. Acostumbro ver el lado favorable de las co-

$\begin{array}{lll}2.01 & 1.020 \quad .47\end{array}$

sas buenas que tiene el barrio en el que vivo.

7. Vivo con cierto desahogo y bienestar.

$\begin{array}{lll}2.15 & 1.140 \quad .61\end{array}$

8. Puedo decir que soy afortunado por tener la

$\begin{array}{lll}1.86 & 1.035 \quad .72\end{array}$

casa que tengo

9. Me siento tranquilo sobre mi futuro

$\begin{array}{lll}2.33 & 1.207 \quad .75\end{array}$

económico.

10. Creo que mi casa ofrece lo necesario para vivir cómodamente.

$\begin{array}{lll}2.07 & 1.047 \quad .85\end{array}$

11. Mi casa me ofrece un ambiente térmico confortable.

$\begin{array}{lll}2.16 & 1.117 \quad .78\end{array}$

12. Mi situación es relativamente próspera.

$2.22 \quad 1.060 \quad .73$

13. Creo que los materiales con los que está

$2.30 \quad 1.227 \quad .65$

construida mi casa me protegen de la temperatura exterior.

14. Estoy conforme con mi casa tal y como

$\begin{array}{lll}2.39 & 1.222 & .74\end{array}$ está (en relación a material de construcción y equipamiento).

15. En comparación con mis vecinos (personas que viven contiguos a mi casa) yo vivo con cierto desahogo y bienestar.

${ }^{*}$ Pesos factoriales para cada reactivo de la escala.

**Alfa de Cronbach fue utilizado como indicador de confiabilidad. 


\section{Resultados de los modelos de ecuaciones es-} tructurales

Modelo de ecuaciones estructurales de la temporada de verano.

Los resultados de la temporada de verano se ilustran en la figura 1, en ella se muestra la estructura factorial entre el componente de percepción del ambiente térmico al interior de la vivienda y los datos del ambiente físico térmico en el interior de la vivienda sobre el constructo de bienestar psicológico asociado con la vivienda.

El factor o constructo de Percepción del ambiente térmico surgió de manera coherente, ya que se produjeron pesos factoriales altos $\mathrm{y}$ significativos $(p<.05)$ para sus variables observadas: Sensación térmica, con un peso factorial (PF) de -.99, Preferencia de ventilación ( $\mathrm{PF}=$ -.34) y Sensación de humedad ( $\mathrm{PF}=.35)$.

La misma situación ocurrió para los factores Ambiente físico térmico y Bienestar psicológico, los cuales quedaron integrados de la siguiente manera, para el factor Ambiente físico térmico: sus variables observadas de Temperatura de bulbo seco $(\mathrm{PF}=.92)$, Temperatura de bulbo húmedo $(\mathrm{PF}=.50)$ y Temperatura de globo, $(\mathrm{PF}=.99)$. El factor bienestar psicológico quedó integrado por Dominio del entorno $(\mathrm{PF}=$ $.99)$, Bienestar material $(\mathrm{PF}=.59)$ y Bienestar subjetivo $(\mathrm{PF}=.58)$.

Se observa, además, una relación negativa y significativa entre el factor de primer orden Ambiente físico térmico sobre el factor Bienestar psicológico, lo cual puede ser un indicativo de que a mayor temperatura hay menos bienestar. Podemos observar, también, una relación positiva y significativa entre el Ambiente físico térmico y la Percepción del ambiente térmico. Se encontró una relación no significativa entre este último factor y el Bienestar psicológico.

Los indicadores de bondad de ajuste muestran que el modelo está respaldado por los datos (Bentler, 2006). El valor de la $\chi 2$ fue de 19.356 con 19 grados de libertad, asociado a una probabilidad de .43; los indicadores prácticos de bondad de ajuste resultantes fueron: BentlerBonett Index or Normed Fit Index (NFI) de .99, Bentler-Bonett Non-Normed Fit Index (NNFI) de .99, Comparative Fit Index (CFI) de .99. El grado de error del modelo se midió mediante el error de aproximación de la raíz media cuadrada (RMSEA, por sus siglas en inglés). Valores alrededor de .05 indican un muy buen ajuste del modelo, aunque valores por debajo de .10 se consideran como aceptables, el RMSEA para este modelo fue .012 . 


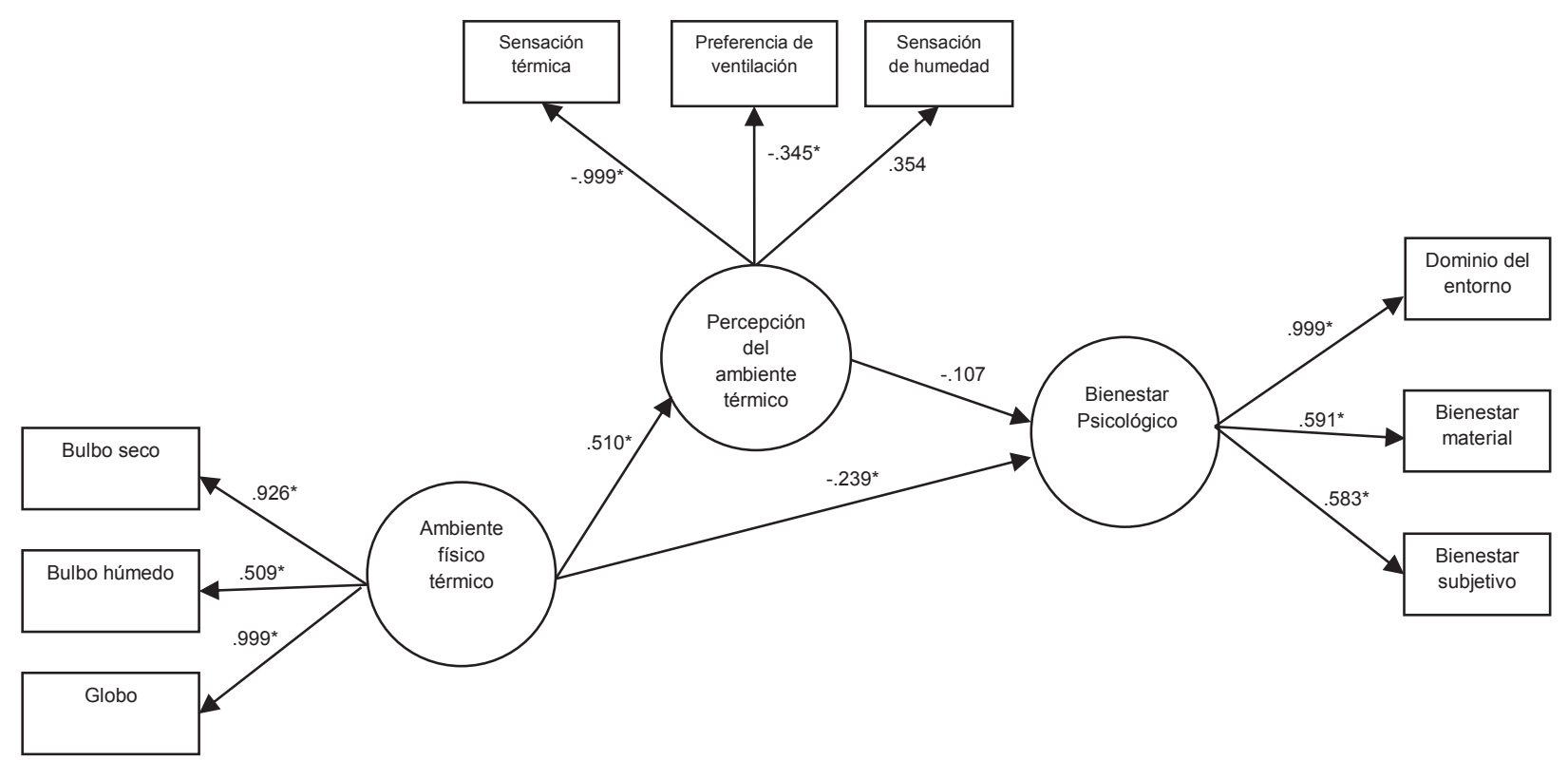

Figura 1. Modelo de bienestar psicológico, percepción del ambiente térmico y ambiente físico térmico en habitantes de viviendas de interés social en clima cálido-seco (verano).

\section{Modelo de ecuaciones estructurales de la} temporada invernal.

Los resultados de la temporada de invierno se muestran en la figura 2, en ella se observa la estructura factorial entre el componente de Percepción del ambiente térmico al interior de la vivienda y los datos del Ambiente físico térmico en el interior de la vivienda sobre el constructo de Bienestar psicológico asociado a la vivienda.

El factor o constructo de Percepción del ambiente térmico surgió de manera coherente, pues se produjeron pesos factoriales altos y significativos $(p<.05)$ para sus variables observadas: Sensación térmica, con un peso factorial (PF) de .74, Preferencia de ventilación $(\mathrm{PF}=.63)$ y Sensación de humedad $(\mathrm{PF}=.27)$.
La misma situación ocurrió para los factores de Ambiente físico térmico y Bienestar psicológico, los cuales quedaron integrados de la siguiente manera para el factor Ambiente físico térmico: sus variables observadas de Temperatura de bulbo seco $(\mathrm{PF}=.90)$, Temperatura de bulbo húmedo $(\mathrm{PF}=.63)$ y Temperatura de globo $(\mathrm{PF}=.94)$. A diferencia del modelo de verano, en el presente modelo se integró el índice de relaciones personales en el factor de Bienestar psicológico, debido a que su inclusión favorecía los índices de bondad de ajuste del modelo; el factor Bienestar psicológico quedó integrado por Dominio del entorno $(\mathrm{PF}=.81)$, Relaciones Personales $(\mathrm{PF}=.63)$, Bienestar material $(\mathrm{PF}=$ $.81)$ y Bienestar subjetivo $(\mathrm{PF}=.80)$. 
Los indicadores de bondad de ajuste muestran que el modelo está respaldado por los datos. El valor de la $\chi 2$ fue de 29.0 con 32 grados de libertad, asociado a una probabilidad de .62; los indicadores prácticos de bondad de ajuste resultantes fueron: Bentler-Bonett Index Normed Fit Index (NFI) de .94, BentlerBonett Non-Normed Fit Index (NNFI) de .99,
Comparative Fit Index (CFI) de .99 y el RMSEA fue igual a .000 .

A diferencia del modelo anterior, sólo se encontró una relación significativa entre el Ambiente físico térmico y la Percepción del ambiente térmico. No se observó otro tipo de relaciones significativas entre el resto de los factores de primer orden para este modelo.

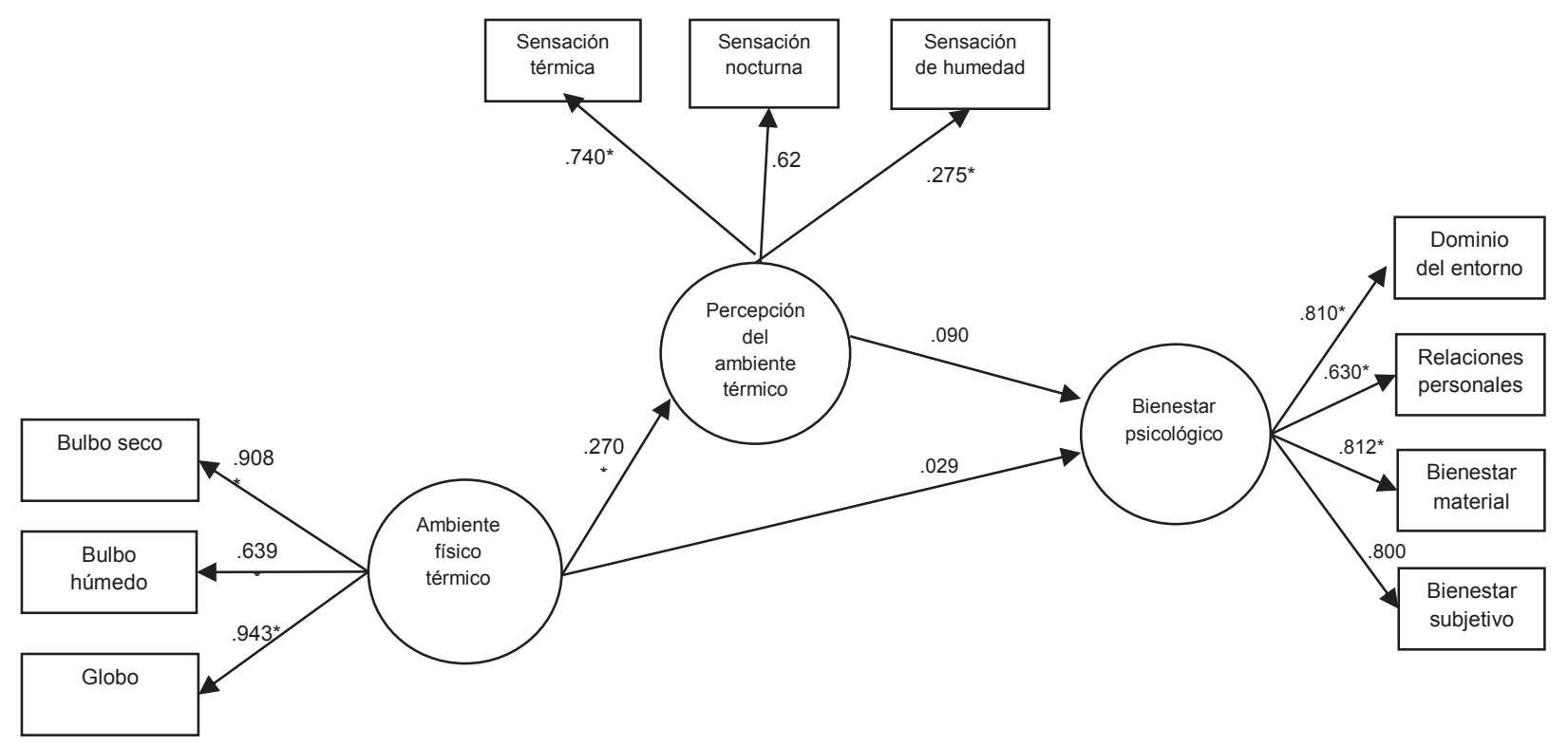

Figura 2. Modelo de bienestar psicológico, percepción del ambiente térmico y ambiente físico térmico en habitantes de viviendas de interés social en clima cálido-seco (invierno).

\section{Discusión}

Aun cuando en la mayor parte del territorio mexicano predominan los climas cálidos, clasificados en subhúmedos y húmedos, además de los climas secos y muy seco, y de la existencia de grandes centros urbanos en zonas donde se presentan altas temperaturas $y$, en el caso de las costas, adicionalmente una alta humedad, el plan nacional de desarrollo urbano y de vivienda 2013-2018 no contempla la generación 
de viviendas con diseños particulares, que se adecuen a las características propias de los climas de cada estado, a la radiación solar y al comportamiento climático de cada región.

Existe una tendencia a prestar atención casi exclusivamente al tamaño de las viviendas como sinónimo de calidad. Si bien es algo comprobado que los espacios habitacionales reducidos tienden a generar la aparición de violencia intrafamiliar, alteraciones en el estado de ánimo, abuso sexual y generación de múltiples circuitos de agresividad (Lotito, 2009), es necesario recordar la presencia de otros factores que pueden afectar la calidad de la vivienda y, por lo tanto, la calidad de vida de las personas que la habitan.

La calidad de vida se ha convertido en un término utilizado con frecuencia en contextos muy variados tanto políticos, como psicológicos, económicos, médicos y educativos. GarcíaViniegras (2005) menciona que la calidad de vida se encuentra altamente relacionada con el bienestar psicológico, como una categoría de la calidad de vida; según la autora, el bienestar psicológico se genera como resultado de un juicio de satisfacción con la vida (balance entre expectativa y logros) en las áreas más significativas para la persona.

Para este estudio, se abordó la variable de bienestar psicológico con base en cuatro dimensiones: las relaciones personales, el dominio del entorno, el bienestar subjetivo y el bienestar material. Lo anterior implica que las personas que viven en estas viviendas de interés social en clima cálido-seco, donde la temperatura me- dia encontrada al interior fue de 34.1, la máxima fue de 43 y la mínima de 28, ven afectada su capacidad de mantener relaciones positivas con otras personas, característica fundamental del funcionamiento psicológico positivo. Asimismo, sufre impactos negativos en su dominio del entorno, relacionado con la habilidad de las personas para elegir o crear entornos favorables para satisfacer los deseos y necesidades propios; ésta es otra de las características del funcionamiento positivo del ser humano, ya que las personas con un alto dominio del entorno poseen una mayor sensación de control sobre el mundo y se sienten capaces de influir sobre el contexto que les rodea.

Este trabajo aporta evidencia empírica, según la cual las condiciones térmicas de las viviendas tienen un efecto negativo en el bienestar psicológico de los usuarios, en la época del año cuando se presentan las temperaturas más altas. Esta relación negativa entre dichas variables nos afirma que hay una tendencia a considerar una sensación de menor bienestar psicológico en las temporadas de verano. Los resultados de los modelos de ecuaciones estructurales de verano e invierno, muestran que las relaciones establecidas como hipótesis al inicio de este proyecto, encuentran sustento con los datos obtenidos de la realidad, mediante los autoreportes de los sujetos entrevistados en sus viviendas y las mediciones de las condiciones físicas de las mismas. Lo anterior se puede afirmar, dado que los modelos produjeron indicadores adecuados de bondad de ajuste, 
tanto estadísticos como prácticos. Tanto para la temporada de verano como para la de invierno, no se encontró una relación significativa de la percepción ambiental en el bienestar psicológico. El ambiente físico térmico afecta el bienestar psicológico de las personas en la temporada de verano, por consecuencia, se observó una relación significativa entre estos factores. No sucedió lo mismo con la temporada invernal, ya que aquí no se registró este efecto.

La política habitacional debería partir de un concepto más amplio e integral de vivienda, el cual incluya en su concepción, además de la vivienda en sí, el entorno urbano que la rodea, la ubicación geográfica, el clima, el diseño de los espacios, la salud ambiental, la calidad de los materiales utilizados, los espacios de relajación o zonas verdes que hay en una comunidad, así como también los aspectos emocionales y los valores personales que las personas les asignamos a los espacios que habitamos.

El término de vivienda digna debería ser concebido como un todo y no sólo hacer referencia a los materiales de construcción o a los servicios que tiene como indicadores fundamentales de lo que debe ser una vivienda digna, en este sentido, la población estará en posibilidades de construir identidades y generar sentimientos de arraigo y de pertenencia con su ciudad.

El transitar hacia un modelo de desarrollo urbano "sustentable" e "inteligente", debería no sólo hacer referencia a la necesidad de frenar la tendencia de las ciudades a crecer de manera desordenada y sin planeación integral, sino que, además, debería buscar el bienestar y la seguridad de sus habitantes.

A pesar de las características climatológicas del país, mencionadas en párrafos anteriores, existen muy pocos estudios que señalen la pertinencia de buscar estrategias para lograr confort térmico en viviendas de interés social; adicionalmente, en los planes de gobierno no hay indicios de querer abordar esta problemática. Lionel y Olivera (2013) señalan que para los programas de vivienda federal y estatal esta visión representaría un cambio de paradigma de viviendas sociales tipo, ya que implica repensar en diseños particulares adecuados a las características propias del clima, la radiación solar y el comportamiento climático de cada región. Esta percepción de no inmediatez en la gratificación por el recurso económico invertido es una problemática que enfrentan todos los problemas de cuidado del medio ambiente en nuestro país.

Podemos mencionar que existen alternativas para mejorar las condiciones térmicas de las viviendas en las entidades que así lo requieran, una de ellas es la arquitectura bioclimática que tiene por objetivo la construcción y diseño sustentable de edificios. En México, existen valiosos esfuerzos de investigación, específicamente en la Universidad de Sonora (UNISON), donde investigadores de la escuela de arquitectura han desarrollado un modelo de vivienda de interés social que se ajusta al clima cálido-seco con materiales que tienen como objetivo conservar una temperatura agradable y con ello mejorar la 
calidad de vida de sus habitantes: utilizando el adobe estabilizado y bloques de concreto celular que reducen el calentamiento de la casa; a diferencia de los que tradicionalmente se utilizan para construir viviendas en Sonora y otras partes de México: materiales industrializados como block o vigueta y bovedilla cuya función es conservar la temperatura.

Aunque a nosotros nos suene extraño, en el ámbito internacional, países como Australia, Canadá y Reino Unido, han establecido como objetivo de política nacional lograr el bienestar subjetivo de sus habitantes, más extraño aún, el Reino de Bután, en el Himalaya, ha declarado que su meta oficial no es ya el crecimiento económico, sino la "felicidad nacional” (Garned \& Prugh, 2008, citado en Corral, 2010).

Finalmente, en cuanto a las limitaciones del trabajo de investigación se pueden mencionar que es necesario realizar réplicas del estudio en poblaciones diversas, en muestras mayores, y el empleo de técnicas alternativas a los autoreportes, con el fin de verificar la validez de las medidas; esperemos que es presente estudio, cuyo objetivo fue la inclusión de factores de orden psicológico en el diseño de viviendas de interés social y en la medición del confort térmico, con énfasis en la importancia del trabajo interdisciplinario, aporte al desarrollo de nuevos modos de vida en la sociedad mexicana al abrir la posibilidad de considerarlos como punto de partida para especificar y probar modelos de bienestar psicológico.

\section{Referencias}

Amérigo, M. (1995). Satisfacción Residencial. Un análisis psicológico de la vivienda y su entorno. Madrid: Alianza editorial.

Aragonés, J. I., \& Amérigo, M. (2000). Psicología Ambiental. Aspectos conceptuales y metodológicos. En J. Aragonés \& M. Amérigo (Coor.), Psicología Ambiental. Madrid: Pirámide.

Baron, R. (1972). Aggression as a function of ambient temperature and prior anger arousal. Journal of Personality and Social Psychology, 21, 183-189.

Baron, R. (1978). Aggression and heat: The "long hot summer". En A. Baum, S. Valins \& J. E. Singer (Eds.), Advances in Environmental Research (pp. 186-207). Hillsdale, NJ: Lawrence Erlbaum Associates Inc.

Bell, P. A., \& Greene, T. C. (1982). Thermal stress: Physiological, comfort, performance, and social effects of hot and cold environments. En G. E. Evans (Ed.), Environmental Stress (75-104). EUA: Cambridge University Press.

Bell, P. A., \& Fusco, M. E. (1989). Heat and violence in the Dallas field data: Linearity, curvilinearity and heteroscedasticity. Journal of Applied Social Psychology, 19, 1979-1982.

Bentler, P. M. (2006). EQS Structural Equations Program Manual Multivariate Software. California: Encino.

Cassidy, T. (1997). Environmental Psychology. Behavior and experience in context. Birmingham, UK: Psychology Press. 
Cohen-Sánchez, L. (2009). Efectos de la intensidad de la luz en el ser humano y su expresión a través del color de la vestimenta. Anales Universitarios de Etología, 3, 29-35.

Cohn, E. G. (1993). The prediction of police call for service: The influence of weather and temporals variables on rape and domestic violence. Journal of environmental psychology, 13, 71-83.

Corral,V.(2010). Psicologíadelasustentabilidad, un análisis de lo que nos hace pro ecológicos y pro sociales. México: Trillas.

Cuervo, N. (2012). Los avatares de la vivienda en la investigación urbana y regional latinoamericana (1990-2012). Territorios, 27, 4770.

Díaz, D., Rodríguez-Carvajal, R., Blanco, A., Moreno-Jiménez, B., Gallardo, I., Valle, C., \& van Dierendonck, D. (2006). Adaptación española de las escalas de bienestar psicológico de Ryff. Psicothema, 18, 572-577.

Field, S. (1992). The effect on temperature on crime. British Journal of Criminology, 32, 340-351.

García-Viniegras, C. (2005). El bienestar psicológico: Dimensión subjetiva de la calidad de vida. Revista Electrónica de Psicología Iztacala, 8, 1-20.

Hernández, B. G., \& Gómez A. A. (2007). La temperatura ambiental y su vinculación con el aprovechamiento escolar. Palapa, Revista de Investigación Científica en Arquitectura, 2, 21-30.
Holahan, C. J. (2010). Psicología ambiental un enfoque general. México: Limusa.

Keller, M. C., Fredrickson, B. L., Ybarra, O., Cote, S., Johnson, K., Mikels, J., \& Wager, T. (2005). A warm heart and clear head: The contingent effects of weather on mood and cognition. Psychological Science, 16, 724731.

Lambert, G., Reid, C., Kaye, D., Jennings, G., \& Esler, M. (2003). Increased Suicide Rate in the Middle-Aged and Its Association With Hours of Sunlight. The American Journal of Psychiatry, 160, 793-795.

Lewin, K. (1964). Field Theory in Social Science: Select theoretical papers. New York: Harper and Row.

Lionel, C. L. \& Olivera, S. M. (2013). Evaluación económica de la resistencia térmica de la vivienda de interés social en las ciudades tropicales de México. Acta Universitaria de la Universidad de Guanajuato, 23, 17-29.

Lotito, C. F. (2009). Arquitectura, psicología, espacio e individuo. Aus (Valdivia), 6, 12-17. ISSN 0718-7262.

Ponce, G. (2011). Habitar en México: Calidad y rezago habitacional en la primera década del milenio [Publicado en versión preliminar del documento de trabajo No. 112 del Centro de Estudios Sociales y de Opinión Pública de la Cámara de Diputados, LX Legislatura]. México.

Rim, Y. (1975). Psychological test performance of different personality types on Sharav 
days in artificial air ionization. International Journal of Biometeorology, 21, 337-340.

Rosen, S. (1985). The weather: Wendy and grounchy. The Catholic Digest, 94-97.

Rosenthal, N. E. (1993). Winter blues. New York: Guilford Press.

Sánchez, C. J. (1998). Escala de Bienestar Psicológico. Madrid: TEA Ediciones. 